\title{
Microbiological Profile of Infections in a Tertiary Care Burns Unit
}

\author{
Ebenezer $\mathrm{R}^{1}$, Isabella Princess ${ }^{2}$, Rohit Vadala ${ }^{3}$, Suresh Kumar ${ }^{4}$, Nagarajan Ramakrishnan ${ }^{5}$, Ganapathy Krishnan ${ }^{6}$
}

\begin{abstract} tertiary care hospital. should be planned based on culture reports.

Indian Journal of Critical Care Medicine (2019): 10.5005/jp-journals-10071-23234

\section{INTRODUCTION}

Infections are one of the most common complication encountered in patients hospitalized with severe burns. Mortality due to infections is alarming as most of them are caused by multidrug resistant bugs harbored in the hospital environment. ${ }^{1}$ It is therefore mandatory to study the etiology of various infections, antibiotic susceptibility pattern of microorganisms isolated from clinical samples. Antibiotic policy of burns units should be based on local antibiogram in order to specifically target common organisms isolated from these patients.
\end{abstract}

Background: The burden of infections among burns patients is higher in healthcare settings due to partial or complete loss of skin as a physical barrier among these patients. We intend to present microbiological profile of patients admitted to a tertiary care hospital in South India.

Aim: To describe microbiological profile of infections and antimicrobial susceptibility pattern of clinical isolates from burns patients in our

Materials and methods: This retrospective analysis was done on consecutive patients admitted with burns over a period of three years at Apollo Specialty Hospitals, a tertiary care facility in Vanagaram, Chennai. Data analysis included clinical isolates from blood, urine, tissue, pus and tracheal aspirate. Types of bloodstream infections, urosepsis and antibiogram are described.

Results: Among 219 clinical isolates from various samples, 75\% were gram-negative, 19\% gram-positive and 6\% were yeast like fungi. Among bloodstream infections, 32\% were polymicrobial. Urosepsis was observed in 39\% patients. Wound infections with sepsis was seen in $39 \%$ patients. Gram-negative isolates showed better susceptibility to amikacin, carbapenems, beta lactam - beta lactamase inhibitor combinations. Gram-positive isolates had better susceptibility to macrolides, doxycycline, glycopeptides.

Conclusion: The high prevalence of gram-negative, polymicrobial infections and multidrug resistant bacteria noted in our patients and the sensitivity patterns would help with appropriate decision on initial antibiotic therapy. However escalation and de-escalation of antibiotics

Keywords: Antibiogram, bloodstream infection, burn wound infection, burns infection, polymicrobial infection

\section{Materials and Methods}

This retrospective analysis was carried out by analyzing data collected over a period of three years (2014 to 2017) from all burns patients admitted to our tertiary care hospital in Chennai, Tamil Nadu, India. Clinical details and microbial profile were collected and documented.

Microbial profile analyzed included the following types of microorganisms and common organisms isolated from various clinical samples, types of bloodstream infections in burns and mortality rate in bloodstream infections, urosepsis and wound infections predisposing to sepsis, antibiogram of gram-negative bacilli, multidrug resistant gram-negative bacilli and gram-positive cocci. Difference in pattern of organisms isolated within and more than 48 hours of admission among direct admissions and patients referred from other hospitals were also analyzed.

All samples collected from burns patients were transported immediately and processed in the microbiology laboratory.

\footnotetext{
1,3,5 Department of Critical Care Medicine, Apollo Specialty Hospitals, Vanagaram, Chennai, Tamil Nadu, India

${ }^{2}$ Department of Microbiology, Apollo Specialty Hospitals, Vanagaram, Chennai, Tamil Nadu, India

${ }^{4}$ Department of Infectious Diseases, Apollo Specialty Hospitals, Vanagaram, Chennai, Tamil Nadu, India

${ }^{6}$ Department of Plastic Surgery, Apollo Specialty Hospitals, Vanagaram, Chennai, Tamil Nadu, India

Corresponding Author: Isabella Princess, Department of Microbiology, Apollo Specialty Hospitals, Vanagaram, Chennai, Tamil Nadu, India, Phone: 9941012080, e-mail: drisabella_p@apollohospitals.com

How to cite this article: Ebenezer R, Princess I, Vadala R, Kumar S, Ramakrishnan N, Krishnan G. Microbiological Profile of Infections in a Tertiary Care Burns Unit. Indian J Crit Care Med 2019;23(9):405-410.

Source of support: Nil

Conflict of interest: None
}

Processing of clinical samples were done based on the department's Standard Operating Protocol. Significant clinical isolates were included based on colony morphology on blood agar, MacConkey agar, chocolate agar. Standard biochemical tests were performed for identification of these isolates. Nonfermenting gram-negative bacilli were identified using Vitek-2 compact system. Antimicrobial susceptibility testing was performed using Kirby Bauer disk diffusion method and Minimum Inhibitory Concentration for second line drugs were performed using E strips. All data were collected and documented in Microsoft Excel software for analysis.

(c) The Author(s). 2019 Open Access This article is distributed under the terms of the Creative Commons Attribution 4.0 International License (https://creativecommons. org/licenses/by-nc/4.0/), which permits unrestricted use, distribution, and non-commercial reproduction in any medium, provided you give appropriate credit to the original author(s) and the source, provide a link to the Creative Commons license, and indicate if changes were made. The Creative Commons Public Domain Dedication waiver (http://creativecommons.org/publicdomain/zero/1.0/) applies to the data made available in this article, unless otherwise stated. 


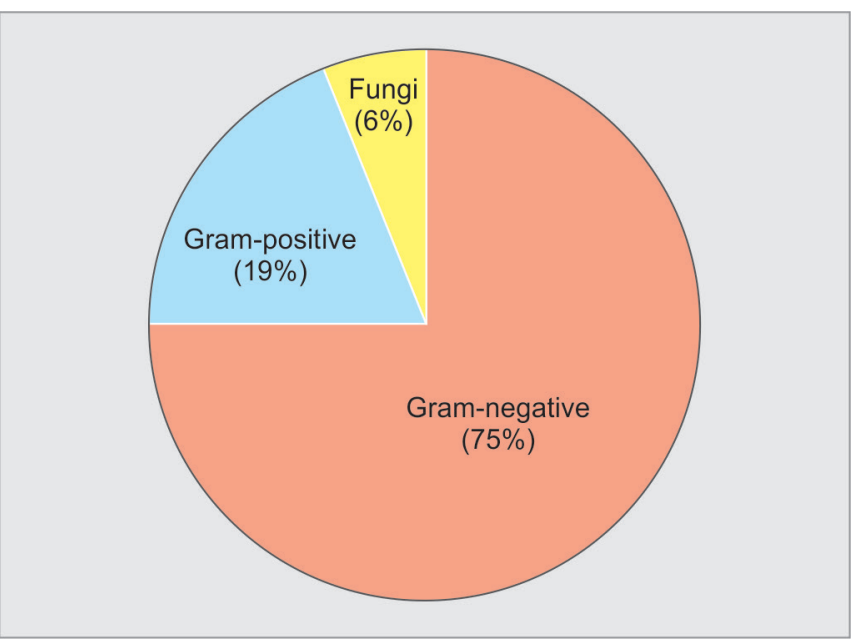

Fig. 1: Distribution of organisms isolated from burns patients $(n=219)$

\section{Results}

Among 94 burns patients admitted over a period of three years, 219 clinically significant microorganisms were isolated. Distribution of the types of organisms is illustrated in Figure 1. 75\% isolates were gram-negative bacilli, among which 59\% were multidrug resistant. Remaining $25 \%$ isolates were constituted by gram-positive cocci (19\%) and yeast like fungi (6\%). Filamentous fungi were not isolated from any patient.

Organisms isolated from blood were predominantly gramnegative bacilli (73\%) followed by gram-positive cocci (24\%) and yeast like fungi (3\%). The pattern was different among urine isolates where gram-negative bacilli were still predominant but to a lesser extent (52\%), followed by yeast like fungi (38\%) and then grampositive cocci (10\%). Of note, yeast like fungi were not isolated from

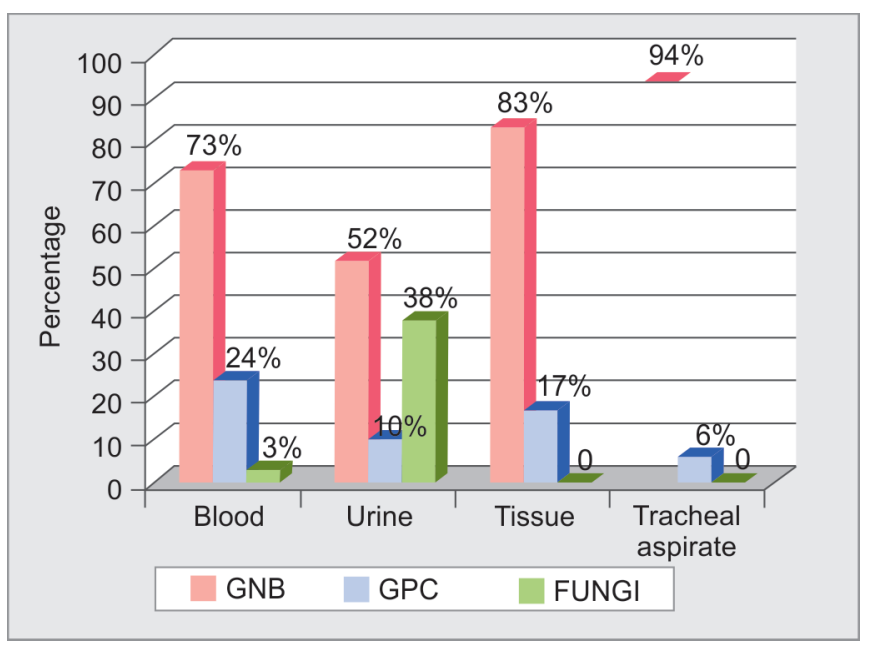

Fig. 2: Distribution of organisms isolated from various samples of burns patients $(n=219)$

tracheal aspirates and tissue/pus isolates. Percentage distribution of types of organisms among various samples is illustrated in Figure 2.

Among gram-negative organisms, the most common were Pseudomonas, Klebsiella and Acinetobacter. Enterococcus was the common gram-positive isolate and Candida species being the most common yeast like fungi. A detailed distribution of organisms isolated from various clinical samples is elicited in Table 1.

A $72.5 \%$ with severe burns developed sepsis. Polymicrobial bloodstream infections were seen in $68 \%$ and monomicrobial infections in $32 \%$ patients (Fig. 3). Among polymicrobial bloodstream infections, combinations of gram-positive cocci with a gram-negative bacilli was seen in $56 \%$, combinations of more than one gram-negative organism was seen in $38 \%$, and $6 \%$ patients had a combination of gram-positive cocci, gram-negative bacilli and yeast like fungi.

Table 1: Concordance of clinical isolates from various samples of burns patients $(n=219)$

\begin{tabular}{|c|c|c|c|c|}
\hline Type of organism & Blood & $\begin{array}{l}\text { Tissue/Pus/ } \\
\text { Wound } \\
\text { swab }\end{array}$ & Urine & $\begin{array}{l}\text { Tracheal } \\
\text { aspirate }\end{array}$ \\
\hline \multicolumn{5}{|l|}{ Gram-negative bacilli } \\
\hline - Pseudomonas species & 23 & 19 & 1 & 6 \\
\hline • Klebsiella species & 22 & 18 & 6 & 2 \\
\hline - Acinetobacter species & 13 & 9 & 1 & 7 \\
\hline - Proteus mirabilis & 2 & 7 & 1 & - \\
\hline - NFGNB & 6 & 1 & 1 & - \\
\hline $\begin{array}{l}\text { - Others (E.coli, Enterobacter, } \\
\text { Citrobacter) }\end{array}$ & 10 & 3 & 5 & 1 \\
\hline Total gram-negatives : 164 & 76 & 57 & 15 & 16 \\
\hline \multicolumn{5}{|l|}{ Gram positive cocci } \\
\hline - Enterococcus species & 14 & 6 & 3 & - \\
\hline - MRCoNS & 7 & 2 & - & 1 \\
\hline - MRSA & 2 & 1 & - & - \\
\hline - Streptococcus species & 2 & 1 & - & - \\
\hline - MSSA & - & 2 & - & - \\
\hline Total gram-positives : 41 & 25 & 12 & 3 & 1 \\
\hline \multicolumn{5}{|l|}{ Fungi } \\
\hline - Candida species & 3 & - & 9 & - \\
\hline - Trichosporon & - & - & 2 & - \\
\hline Total Fungi : 14 & 3 & - & 11 & - \\
\hline Total isolates : 219 & 104 & 69 & 29 & 17 \\
\hline
\end{tabular}




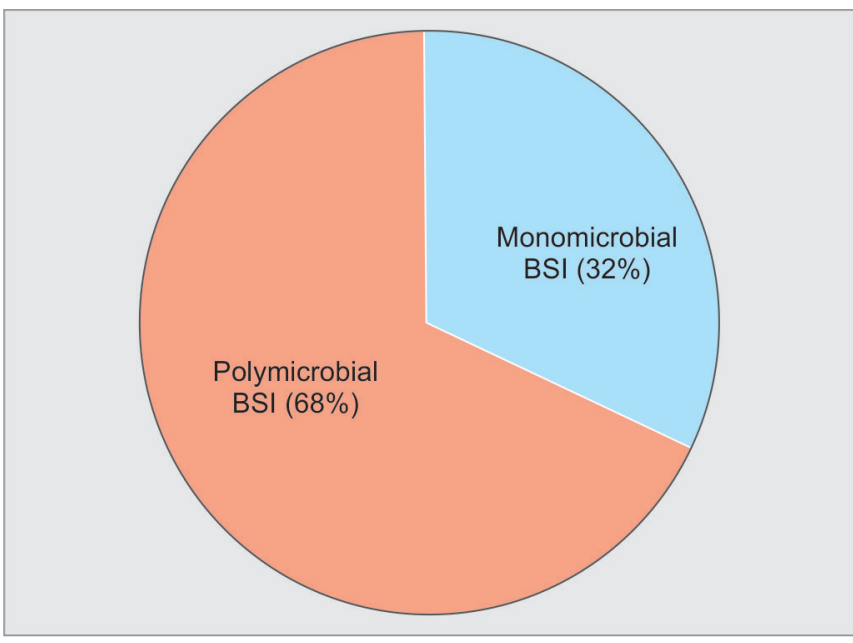

Fig. 3: Distribution of type of bloodstream infections in burns patients

A mortality rate of $59 \%$ was observed in patients with polymicrobial bloodstream infections. A lesser mortality rate of $41 \%$ was seen in patients with monomicrobial bloodstream infections.

Urosepsis was seen in 39\% patients, the most common organism causing the same being Klebsiella species. Wound infection related sepsis was seen in another $39 \%$ of burns patients, the most common organism being Pseudomonas species.

The profile of infections was different based on whether it was early (48 hours after admission) or later. It was also noted that the isolates were different among patients referred from outside hospitals in comparison with those directly admitted to our facility (Table 2).

Antibiogram of isolates showed that less than $50 \%$ of gramnegative isolates were susceptible to gentamicin (40\%), cefotaxime (42\%), ceftazidime (44\%), ciprofloxacin (33\%), and cefepime (39\%). Morethan $50 \%$ of gram-negatives were susceptible to amikacin (53\%), cefoperazone-sulbactam (56\%), piperacillin-tazobactam (54\%), imipenem (59\%), meropenem (60\%) and ertapenem (80\%) (Fig. 4).

Susceptibility to fosfomycin, colistin and polymyxin B was seen in $96 \%, 100 \%$ and $100 \%$ isolates, respectively (Fig. 5).

Considerably lower rate of resistance was seen among grampositive clinical isolates. Less than $50 \%$ of gram-positive organisms were susceptible to penicillin (25\%) and clindamycin (43\%). All other antibiotics showed good percentage of action against grampositive cocci (Fig. 6).

\section{Discussion}

Mortality rate due to infections is high among adult and pediatric burns patients in India and other developed countries. ${ }^{2,3}$ Organisms

Table 2: Distribution of isolates based on time and place of admission

\begin{tabular}{|c|c|}
\hline \multicolumn{2}{|c|}{$\leq 48$ hours of admission } \\
\hline Direct admission & Referred from other hospitals \\
\hline $\begin{array}{l}\text { Gram-negative bacilli: } \\
\text { - Pseudomonas aeruginosa (25\%) } \\
\text { Acinetobacter baumannii (25\%) } \\
\text { - Klebsiella pneumoniae (25\%) Enterococcus } \\
\text { species (25\%) }\end{array}$ & 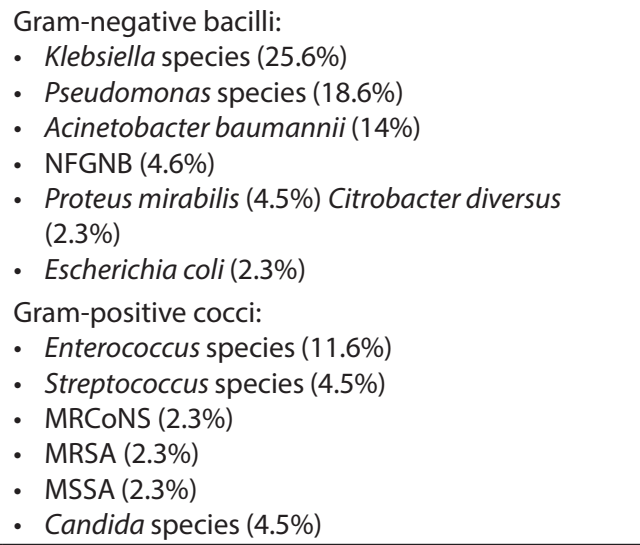 \\
\hline \multicolumn{2}{|c|}{$>48$ hours of admission } \\
\hline Direct admission & Referred from other hospitals \\
\hline $\begin{array}{l}\text { Gram-negative bacilli: } \\
\text { - Pseudomonas species (26.4\%) } \\
\text { - Acinetobacter baumannii (13.2\%) } \\
\text { - Klebsiella species (13.2\%) } \\
\text { - Proteus mirabilis }(5.7 \%) \\
\text { - Escherichia coli }(5.7 \%) \text { NFGNB (3.8\%) } \\
\text { - Enterobacter species (1.9\%) }\end{array}$ & 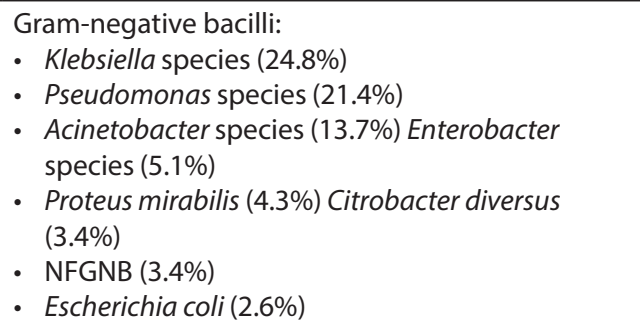 \\
\hline $\begin{array}{l}\text { Gram-positive cocci: } \\
\text { - } \quad \text { Enterococcus species (13.2\%) } \\
\text { - } \text { MRCoNS (3.8\%) } \\
\text { - } \quad \text { MRSA (3.8\%) }\end{array}$ & $\begin{array}{l}\text { Gram positive cocci: } \\
\text { - Enterococcus species ( } 8.5 \%) \\
\text { - MRCoNS (6\%) Streptococcus species (0.9\%) MSSA } \\
(0.9 \%)\end{array}$ \\
\hline Candida species (9.4\%) & $\begin{array}{l}\text { Candida species (3.4\%) } \\
\text { Trichosporon species (1.7\%) }\end{array}$ \\
\hline
\end{tabular}




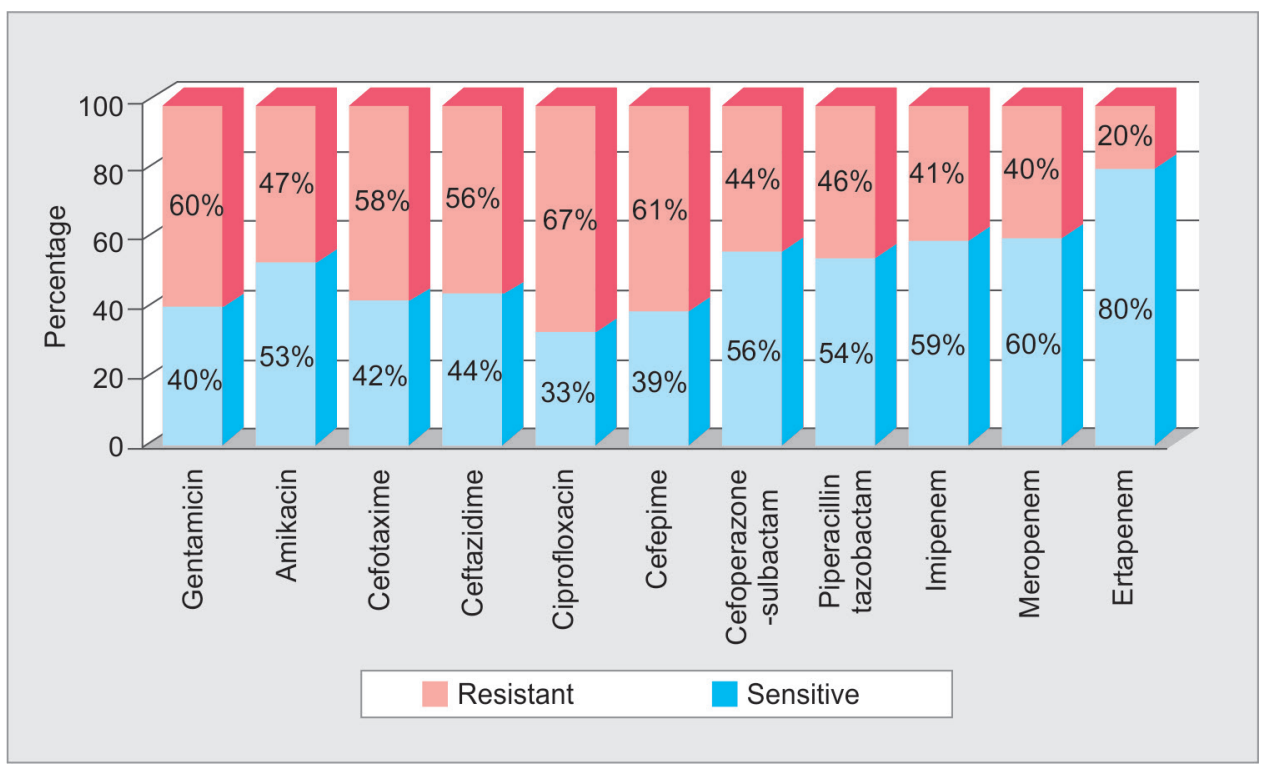

Fig. 4: Percentage of sensitivity and resistance of gram-negative isolates from burns patients $(n=164)$

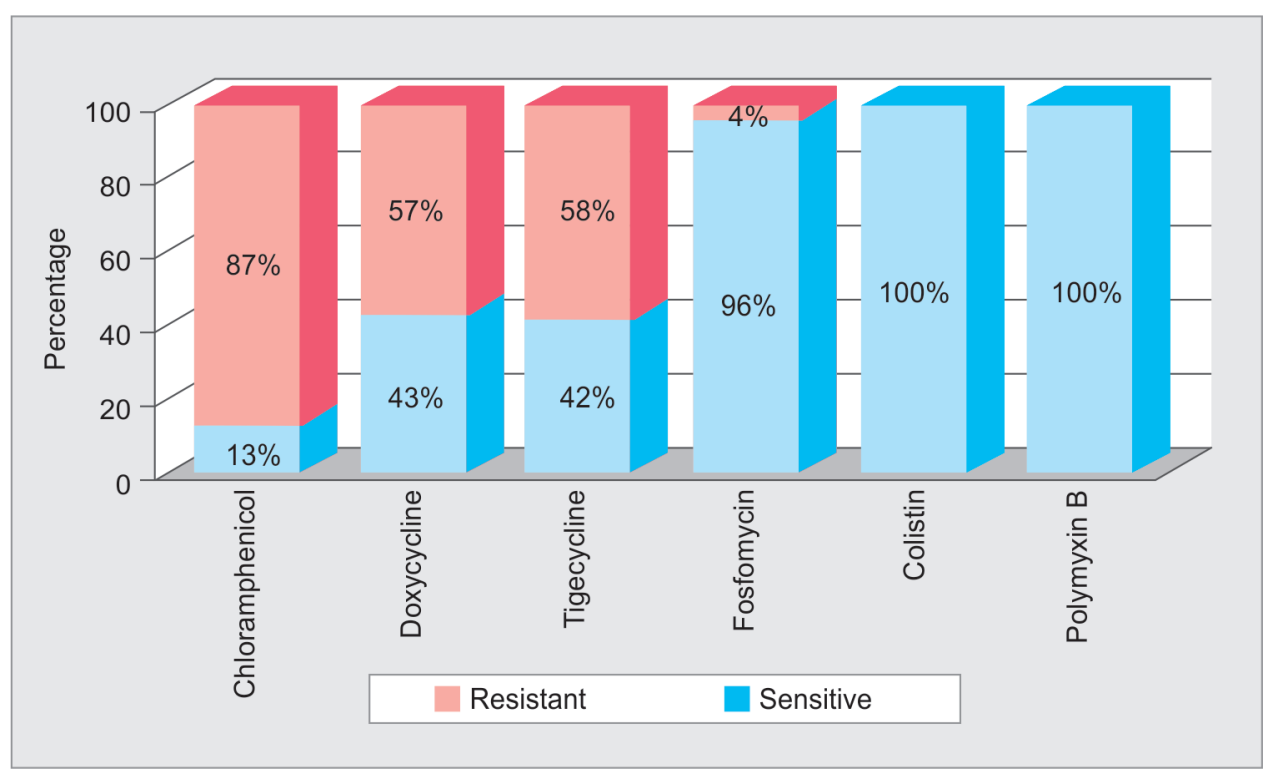

Fig. 5: Percentage of sensitivity and resistance to second-line drugs of MDR gram-negative isolates from burns patients

causing infections in burns patients gain their access through colonization of the host as primary barrier to the organisms is lost. ${ }^{4}$ Therefore, microorganisms present in the hospital environment, patient's own resident flora or a combination of both become the usual causative factors of infection. ${ }^{1}$ Organism profile and their susceptibility to antibiotics vary between every hospital and geographic location. For this reason, we carried out this study among burns patients to analyze types of organisms and their antibiogram.

Gram-negative infections were predominant among burns patients, being consistent with data from a large meta analysis published in 2014. ${ }^{5}$ Among gram-negatives, Pseudomonas species and Klebsiella species top the list of causative pathogens. Similar kind of preponderance to these organisms were observed in two studies from Maharashtra ${ }^{6,7}$ and one from Karnataka. ${ }^{8}$ From our study as well as the literature reviewed by us, it is proven that gram-negative infections due to Pseudomonas, Klebsiella and Acinetobacter are common among burns patients.

On the other hand, incidence of gram-positive infections among our burn patients was 19\%, being much lower than other studies from central India $(26 \%)^{9}$ and China $(31.3 \%) .{ }^{10}$ gram-positive organisms were common in bloodstream infections, out of which $99 \%$ were associated with polymicrobial bloodstream infections. Enterococcus species was the most common gram-positive organism isolated (11\% incidence). We did not encounter vancomycin resistant enterococci from any clinical sample. One contrasting finding in our study was the very negligible percentage of Staphylococcus aureus (MSSA and MRSA) infections. This finding was contrary to various studies from India and Iran. ${ }^{6,8,11}$ Other gram-positives such as MRSA and coagulase negative staphylococci were isolated from a meager number of clinical samples. Studies so far have reported contradicting findings with high incidence of MRSA, MSSA from 


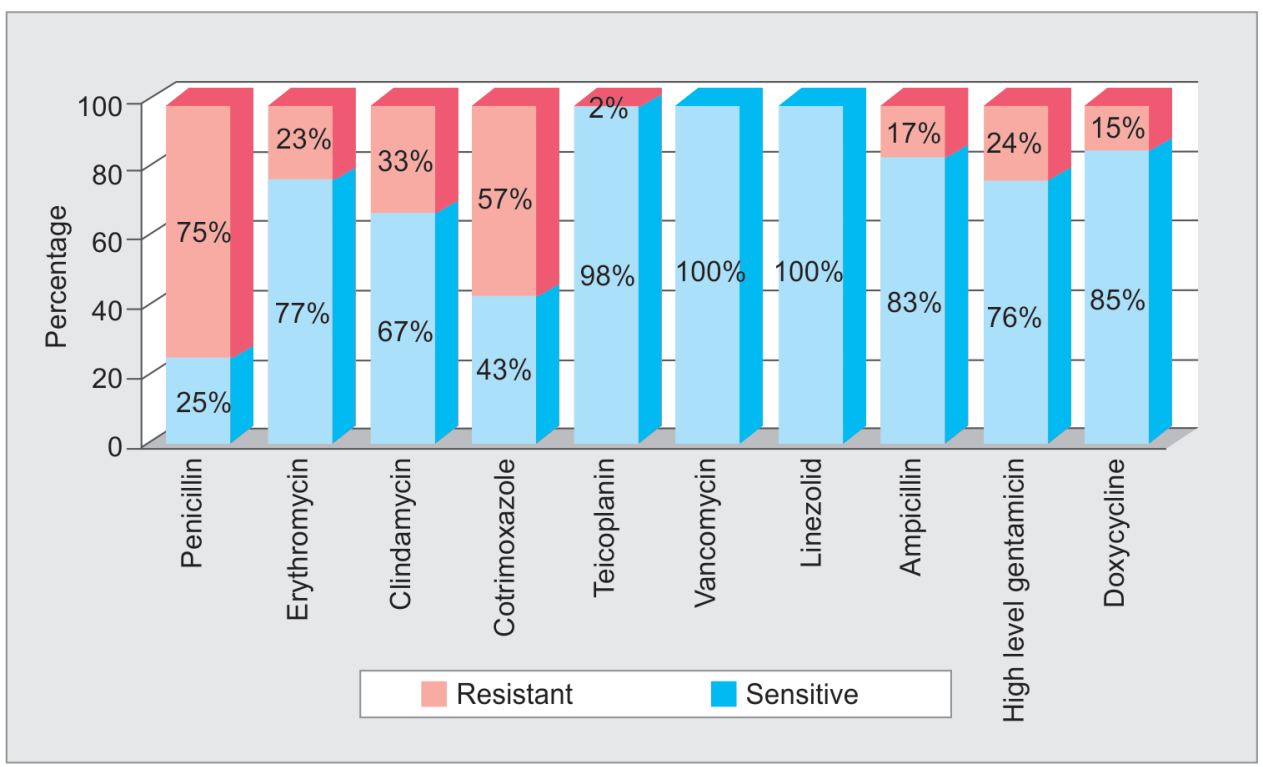

Fig. 6: Percentage of sensitivity and resistance of Gram-positive isolates from burns patients

burns patients. The same studies have not reported enterococcal infections from burns patients. ${ }^{12,13}$ Among drug resistant pathogens in burns patients, MRSA is not therefore a major threat in our center (1.4\% incidence).

According to a review on fungal infections in burns patients, standard guidelines have been placed for diagnosis of invasive fungal infections. ${ }^{14}$ Using these standards, a significant colony count for urine isolates and isolation of Candida species in more than one clinical sample were taken into consideration before making a diagnosis. Among our patients, 14 patients had yeast like fungi in blood and/or urine cultures. All patients with invasive candidiasis succumbed to infection (100\% mortality). The duration of stay of patients with candiduria and invasive candidiasis was longer than those without these conditions (mean length of stay: 24.8 days). Fungal burn wound infections and infections due to molds such as Aspergillus and Zygomycetes were not encountered in our study. Good environmental conditions and air quality play a pivotal role in containing mold infections among patients and we were able to achieve the same in our unit. Large scale studies from France and Australia have also shown a low incidence of noncandidal fungal infections in burns patients. ${ }^{15,16}$

Trichosporon species causing infections among burns patients have been reported by very few authors and literature search did not reveal reports from India. ${ }^{17,18}$ Two of our patients had Trichosporon species infection, both isolated from urine as these infections start as colonization of urinary catheters. Trichosporon can thus be considered as an emerging pathogen among burns patients. They have better susceptibility to echinocandins but variable susceptibility to amphotericin B and fluconazole. ${ }^{19}$ Invasive infection with Trichosporon species may be fatal, therefore isolation from urine cannot be ignored as a colonizer.

Urosepsis among burns patients is a gray zone as this has not been an area of research among these group of patients. Among our patients with sepsis, 10 patients had same organisms isolated from urine and blood (39\% incidence). Growth in urine culture can thus be used to predict or anticipate similar organisms in blood in case of development of sepsis. Another interesting finding was that there were no gram-positive organisms causing urosepsis.

Similarly, only gram-negative organisms were isolated from wound infections as well as blood of 13 patients. No gram-positive organisms were associated with wound infection predisposing to sepsis. It may therefore be postulated that gram-negative organisms in burns patients get disseminated from their primary infective foci and gram-positive organisms do not get disseminated.

Antibiograms of burn centers should be exclusive and empirical treatment algorithms must be based on an updated yearly antibiogram data. ${ }^{20}$ Based on this study, we would incorporate data from this antibiogram for our future use. An updated antibiogram is emphasized due to change in trends of microorganisms and their susceptibility to various antibiotics. The most common example would be the rise in multi drug resistant gram-negative isolates from 2014 to 2017 in our center as well as other centers. ${ }^{21}$ Keeping this in mind, literature search revealed antibiograms of isolates isolated only from burn wounds. Whereas, our study includes a comprehensive antibiogram of all clinical samples (wound, blood, urine, tissue, respiratory samples). We have compared this with two studies published in 2004 from Uttar Pradesh and in 2014 from Chennai. ${ }^{20,21}$ This comparison showed that our gramnegative clinical isolates had better susceptibility to gentamicin, cefotaxime, ciprofloxacin than other studies. Few antibiotics like carbapenems, cefoperazone sulbactam could not be compared due to unavailability of data from studies in and outside India. However, in our study, $80 \%$ of isolates were susceptible to ertapenem. Ertapenem has low risk of development of resistance when compared to other carbapenems. Better susceptibility was noted among gram-positive isolates from our patients to ampicillin, doxycycline, erythromycin and penicillin when compared to other studies. ${ }^{20,21}$

Large scale studies on microbial profile and susceptibility pattern of burns isolates are required in future. This would provide an insight on predominant resistance patterns and thus help clinicians to make targeted empiric antibiotic therapy for burns patients. 


\section{Conclusion}

Studies published so far have mainly concentrated only on burn wound infections and few on sepsis among burns patients. Our study provides comprehensive microbial profile and their antibiogram from all samples of burns patients which could help guide empiric choice of antibiotics based on suspected source. Efforts will be made to include this susceptibility pattern in our antibiotic policy for future use in these group of patients. This would also be a stepping stone to formulate separate yearly antibiogram in our hospital which serves as a tertiary care referral center for burns.

\section{References}

1. Mohapatra S, Deb M, Agrawal K, Chopra S, Gaind R. Bacteriological profile of patients and environmental samples in burn intensive care unit: A pilot study from a tertiary care hospital. Indian J Burns 2014;22:62-66.

2. Sharma BR, Singh VP, Bangar S, Gupta N. Septicemia: The Principal Killer of Burns Patients. Am J Infect Dis 2005;1:132-138.

3. Williams FN, Herndon DN, Hawkins HK, Lee JO, Cox RA, Kulp GA, et al. The leading causes of death after burn injury in a single pediatric burn center. Crit Care 2009;13:R183 (doi:10.1186/cc8170).

4. Church D, Elsayed S, Reid O, Winston B and Lindsay R. Burn wound infections. Clin Microbiol Rev 2006;19:403-434.

5. Azzopardi EA, Azzopardi E, Camilleri L, Villapalos J, Boyce DE (2014) Gram Negative Wound Infection in Hospitalised Adult Burn Patients-Systematic Review and Metanalysis-. PLoS ONE 9(4): e95042. doi:10.1371/journal.pone.0095042.

6. Mundhada SG, Waghmare PH, Rathod PG, Ingole KV. Bacterial and fungal profile of burn wound infections in Tertiary Care Center. Indian J Burns 2015;23:71-75.

7. Bhatt P, Rathi KR, Hazra S, Sharma A, Shete V. Prevalence of multidrug resistant Pseudomonas aeruginosa infection in burn patients at a tertiary care centre. Indian J Burns 2015;23:56-59.

8. Kulkarni V, Arali SM, Jayaraj YM, Shivannavar CT, Joshi MR. Bacterial etiology and their antibiogram in burn wound infections at Kalaburgi region (India). Indian J Burns 2015;23:65-70.
9. Chamania S, Hemvani N, Joshi S. Burn wound infection: Current problem and unmet needs. Indian J Burns 2012;20:18-22.

10. Lee HG, Jang J, Choi JE, Chung DC, Han JW. Blood stream infections in patients in the burns intensive care unit. Infect Chemother 2013;45(2):194-201.

11. Ekrami A, Kalantar E. Bacterial infections in burns patients. Indian J Med Res 2008;127:415-416.

12. Mill LS, Benedette CEM, Maximo LZ, Almeida PC, Gomes DS, Gempe $\mathrm{R}$, et al. Bloodstream infections by multidrug-resistant bacteria in patients in an intensive care unit for the treatment of burns: a 4-yearexperience. Rev Bras Cir Plást 2012;27(3):374-378.

13. Gupta V, Garg R, Kaur M, Garg S, Attri AK, Chander J. Prevalent resistance mechanisms in isolates from patients with burn wounds. Indian J Burns 2015;23:60-64.

14. Ballard J, Edelman L, Saffle J, Sheridan R, Kagan R, Bracco D, et al. Multicenter Trials Group, American Burn Association. Positive fungal cultures in burn patients: A multicenter review. J Burn Care Res 2008;29:213-221.

15. Katz T, Wasiak J, Cleland H, Padiglone A. Incidence of non-candidal fungal infections in severe burn injury: An Australian perspective. Burns 2014;40: 881-886.

16. Schaal JV, Leclerc T, Soler C, Donat N, Cirrode A, Jault P, et al. Epidemiology of filamentous fungal infections in burned patients: A French retrospective study. Burns 2015; 41: 853-863.

17. Escarrá F, Lema J, Caracciolo B, Carnovale S, Alvarez V, Tramonti N, et al. Trichosporon asahii sepsis associated with urinary catheter in a pediatric burn unit: 2 case reports. Arch Argent Pediatr. 2017;115:311314.

18. Lomas TL, González DM, Luengo Al, Bouza JM, Pérez JM. Nosocomial infection due to Trichosporon asahii in a critical burned patient. Rev Iberoam Micol. 2015;32:257-260.

19. Ribeiro A, Alastruey-Izquierdo A, Gomez-Lopez A, Rodriguez-Tudela $\mathrm{JL}$, Cuenca-Estrella M. Molecular identification and susceptibility testing of Trichosporon isolates from a Brazilian hospital. Rev lberoam Micol. 2008;25:221-225.

20. Leela KV, Ravinder T, Katragadda R, Lavanya K, Chandrasekaran K. Antibiotic sensitivity pattern of bacterial isolates in burns wound infection in a tertiary care hospital. IPP 2016;3:726-729.

21. Chaware Suresh M, Vijay K, Singh AK, Bhatnagar S, Kant SV. Infection in burn wounds: Recent trends of microbial flora and antibiotic sensitivity and resistance. Indian J Burns 2004;12:51-52. 